University of Wollongong

Research Online

Australian Institute for Innovative Materials -

Papers

Australian Institute for Innovative Materials

$1-1-2012$

Parameters controlling emission of terahertz frequency electromagnetic radiation from InAs and GaAs: An ensemble monte carlo simulation study

David Laurence Cortie

University of Wollongong, dlc422@uowmail.edu.au

Roger A. Lewis

University of Wollongong, roger@uow.edu.au

Follow this and additional works at: https://ro.uow.edu.au/aiimpapers

Part of the Engineering Commons, and the Physical Sciences and Mathematics Commons

Research Online is the open access institutional repository for the University of Wollongong. For further information contact the UOW Library: research-pubs@uow.edu.au 


\title{
Parameters controlling emission of terahertz frequency electromagnetic radiation from InAs and GaAs: An ensemble monte carlo simulation study
}

\author{
Abstract \\ The generation, manipulation and detection of terahertz-frequency electromagnetic radiation are topics of \\ great current theoretical and experimental interest. In particular, the coherent generation and detection of \\ ultrashort pulses of terahertz radiation has opened up the field of terahertz time-domain spectroscopy, \\ which permits the simultaneous measurement of amplitude and phase of the terahertz electric field. In \\ this paper, the emission of terahertz radiation from bare semiconductor surfaces following \\ photoexcitation by ultrashort pulses of near-infrared radiation is studied using ensemble Monte Carlo \\ simulations. The simulation allows us to examine separately distinct scattering mechanisms and to vary \\ the materials parameters of bandgap, absorption coefficient, effective mass, and surface potential. The \\ narrower-gap semiconductor InAs and the wider-gap semiconductor GaAs are given as examples in \\ which diffusion and drift processes, respectively, dominate. We find that scattering is relatively \\ unimportant in the case of InAs but is very important in the case of GaAs.
}

\section{Keywords}

study, gaas, simulation, inas, radiation, electromagnetic, frequency, terahertz, emission, controlling, parameters, carlo, monte, ensemble

Disciplines

Engineering | Physical Sciences and Mathematics

\section{Publication Details}

Cortie, D. and Lewis, R. A. (2012). Parameters controlling emission of terahertz frequency electromagnetic radiation from InAs and GaAs: An ensemble monte carlo simulation study. In ECS Transactions, 7-12 October, Honolulu, Hawaii. ECS Transactions, 50 (9), 309-310. 


\title{
Parameters Controlling Emission Of Terahertz Frequency Electromagnetic Radiation From InAs And GaAs: An Ensemble Monte Carlo Simulation Study
}

\author{
D. L. Cortie ${ }^{\mathrm{a}, \mathrm{b}}$ and R. A. Lewis ${ }^{\mathrm{a}, \mathrm{c}}$ \\ a Institute for Superconducting and Electronic Materials, University of Wollongong, \\ Wollongong, New South Wales 2522, Australia \\ ${ }^{\mathrm{b}}$ Present address: The Bragg Institute, Australian Nuclear Science and Technology \\ Organisation, Lucas Heights, New South Wales 2234, Australia. \\ ${ }^{\mathrm{c}}$ Electronic mail: roger@uow.edu.au. URL: http://uow.edu.au/ roger.
}

The generation, manipulation and detection of terahertz-frequency electromagnetic radiation are topics of great current theoretical and experimental interest. In particular, the coherent generation and detection of ultrashort pulses of terahertz radiation has opened up the field of terahertz time-domain spectroscopy, which permits the simultaneous measurement of amplitude and phase of the terahertz electric field. In this paper, the emission of terahertz radiation from bare semiconductor surfaces following photoexcitation by ultrashort pulses of near-infrared radiation is studied using ensemble Monte Carlo simulations. The simulation allows us to examine separately distinct scattering mechanisms and to vary the materials parameters of bandgap, absorption coefficient, effective mass, and surface potential. The narrower-gap semiconductor InAs and the wider-gap semiconductor GaAs are given as examples in which diffusion and drift processes, respectively, dominate. We find that scattering is relatively unimportant in the case of InAs but is very important in the case of GaAs.

\section{Introduction}

The development of methods and understanding in the terahertz $\left(10^{12} \mathrm{~Hz}\right)$ regime has increased rapidly over recent years, as evidenced by the recent review articles (1-3) and books (4-6) published in the area. There are many diverse means of generating terahertzfrequency electromagnetic radiation, including blackbody sources (such as the heated silicon carbide rod known as the globar), optically-pumped molecular lasers, backwardwave oscillators, $p$-Ge in crossed electric and magnetic fields, quantum cascade lasers, frequency difference combination in photo-mixers, non-linear up-conversion, and synchrotrons. Recent developments have been spurred on by the coherent generation and detection of terahertz radiation, usually achieved using as the pump source an ultrashort (sub-ps) laser. The means to convert the pump radiation to the lower frequency terahertz radiation include optical rectification and photoconductivity $(7,8)$. In this paper we examine a simple method to implement, optical excitation of bare semiconductor surfaces. We have previously reported that surface formation itself can give rise to terahertz radiation (9). In this paper we compare and contrast the emission from InAs $(10,11)$ and from GaAs (12) under optical excitation by ultrashort pulses. In our simulations and the results presented here, we use the computational approach described in detail in Ref. 10. 


\section{Results and Discussion}

We find that the excess of laser energy over the bandgap energy plays a key role in the terahertz generation. The greater excess energy in the case of InAs compared to GaAs, for example, results in more energetic photocarriers produced and so a larger resultant terahertz field. In principle, the same effect can be obtained by reducing the bandgap as increasing the pump laser photon energy. The division of the excess energy among the relatively stationary holes and the relatively mobile electrons also is an important factor. For example, reducing the electron effective mass relative to the hole increases the electron initial velocity. As might be expected, a larger optical absorption coefficient leads to a stronger terahertz emission. The absorption coefficient, which is directly related to the absorption depth, also plays a role in the so-called vanguard counterpotential - the phenomenon of early electrons migrating away from the semiconductor surface and setting up a field which repels electrons photocreated later by the laser pulse. The laser pulse length also plays an important role here, with shorter pulses being more efficient in the generation of terahertz radiation. While scattering plays a major role in reducing the terahertz emission from GaAs it has very little effect in InAs; the charge carrier motion may be considered almost collisionless on the timescales involved.

\section{Acknowledgments}

This work was supported by the Australian Research Council, the University of Wollongong, and the Chinese Academy of Sciences Visiting Professorship for Senior International Scientists Grant \#2010T2J07.

\section{References}

1. P. H. Siegel, IEEE Trans. Microwave Theory Tech., 50, 910 (2002).

2. B. Ferguson and X.-C. Zhang, Nature Mater., 1, 26 (2002)

3. R. A. Lewis, Proc. IEEE, 95, 1641 (2007).

4. S. D. Ganichev and W. Prettl, Intense Terahertz Excitation of Semiconductors, Oxford University Press, Oxford (2006).

5. Y.-S. Lee, Principles of Terahertz Science and Technology, Springer, Berlin (2009).

6. X.-C. Zhang and J. Xu, Introduction to THz Wave Photonics, Springer, New York, (2010).

7. S. Hargreaves, L. J. Bignell, R. A. Lewis, D. Schoenherr, M. Sağlam, H. L. Hartnagel, J. Electrochem. Soc., 155, H734 (2008).

8. S. Hargreaves, L. Bignell, R. A. Lewis, D. Schoenherr, M. Sağlam, H. Hartnagel, ECS Trans., 16(40), 87 (2009).

9. D. L. Cortie and R. A. Lewis, Surface Science (accepted for publication, 12 June 2012).

10. D. L. Cortie and R. A. Lewis, Phys. Rev. B, 84, 155328 (2011).

11. D. L. Cortie and R. A. Lewis, Phys. Rev. B, 85, 159901(E) (2012).

12. D. L. Cortie and R. A. Lewis, Appl. Phys. Lett. (accepted for publication, 12 June 2012). 